\title{
Are vending snacks and beverages complying with the Healthier Vending policy across Health Service Executive premises in the Republic of Ireland?
}

\author{
S. Bel-Serrat ${ }^{1}$, I. Stanley ${ }^{1}$, A. Lawless ${ }^{2}$, S. O’Brien ${ }^{2}$, C.C. Kelleher ${ }^{1}$ and C.M. Murrin ${ }^{1}$ \\ ${ }^{1}$ National Nutrition Surveillance Centre, School of Public Health, Physiotherapy and Sports Science, \\ University College Dublin, Dublin, Ireland and ${ }^{2}$ Healthy Eating \& Active Living Programme, Health Service Executive, \\ Dublin, Ireland.
}

In the Republic of Ireland, $39 \%$ and $23 \%$ of the population are overweight and obese, respectively ${ }^{(1)}$. Besides, $35 \%$ consume foods such as sweets, salted snacks or pastries and $16 \%$ drink soft drinks on a daily basis ${ }^{(1)}$. Vending machines typically offer energy-dense and nutrient-poor foods, and contribute to the obesogenic environment with constant exposure and access to unhealthy foods ${ }^{(2)}$. In 2015, the Health Service Executive (HSE) implemented the Healthier Vending Policy to promote healthier food environments by increasing healthier choices and reducing unhealthy choices in vending machines. All products stocked in vending machines under the Policy are required to meet the classification of Better Choice (BC) or Other Choice (OC) products according to specific energy and nutrient criteria (Table 1$)^{(3)}$. The Policy applies to cold soft drinks, confectionery and snacks stocked in all the vending machines located in the HSE premises across the Republic of Ireland ${ }^{(3)}$. We evaluated whether the products stocked by the vending companies complied with the Policy criteria. A list of products stocked in the vending machines was provided by each of the two companies involved in the evaluation. These products were categorised into $\mathrm{BC}$ or $\mathrm{OC}$ using the nutrient values available in the labels of the products and/or on the manufacturers' website and compared against the companies' own classification of BC and OC.

Table 1. Energy and nutrient criteria for products classification into Better Choice or Other Choice according to the HSE Healthier Vending Policy ${ }^{(3)}$.

\begin{tabular}{lll}
\hline & Better Choice $(\mathrm{BC})$ & Other Choice $(\mathrm{OC})$ \\
\hline Energy & $\leq 150 \mathrm{kcal}$ per packet & $\leq 250$ kcal per packet \\
Total Fat & $\leq 20 \mathrm{~g} / 100 \mathrm{~g}$ & Not restricted \\
Saturated Fat & $\leq 5 \mathrm{~g} / 100 \mathrm{~g}$ & Not restricted \\
Sugar & $\leq 15 \mathrm{~g} / 100 \mathrm{~g}$ & Not restricted \\
Salt/Sodium & $\leq 1.5 \mathrm{~g} / 0.6 \mathrm{~g} / 100 \mathrm{~g}$ & Not restricted \\
\hline
\end{tabular}

A total of 117 products were evaluated. Among these, 52 products ( 22 snacks and 30 beverages) were stocked by vending companies as BC products. Thirty-four (65\%) products met the policy criteria. All beverages met the BC criteria whereas only $4(18 \%)$ snacks did. Eighteen products $(35 \%)$, all snacks, did not meet the BC criteria. Although all products, except one, met the energy criterion, they failed to meet the criteria for fat, sugar and/or salt/sodium. Among these, 56\% exceeded one criterion, $22 \%$ exceeded 2 criteria and $22 \%$ exceeded 3 criteria. A total of 65 (51 snacks and 14 beverages) products were stocked by the vending companies as OC products. Most of these products ( $88 \%$ ) met the OC energy criterion. Only eight products ( 7 snacks and 1 beverage) exceeded the energy limit.

In conclusion, while beverages fully complied with the Policy, most of the snacks, mainly BC products, failed to meet the nutrient criteria. These analyses emphasised the current poor availability of healthier snack products in the market. Misclassification of products as BC could lead consumers to misinformed choices and unintentional consumption of less healthier options. Therefore, efforts to increase healthier vending options should be made to promote healthier food environments and consumer choices in these institutions.

1. Healthy Ireland Survey (2017) Department of Health, Dublin.

2. Hua SV, Kimmel L, Van Emmenes M et al. (2017) J Acad Nutr Diet 117, 1057-1065.

3. Health Service Executive - Healthier Vending Policy (2015) Health Service Executive, Dublin 